INVESTIGACIONES

de HISTORIA ECONÓMICA

2007, invierno, número 7. Pp. 133 a 160

\title{
El debate sobre la "Gran Divergencia" y las bases institucionales del desarrollo económico
}

\author{
The debate over the "Great Divergence" and the institutional basis \\ of economic development
}

\author{
ENRIQUE IBÁÑEZ ROJO \\ Universidad Rey Juan Carlos, Madrid
}

\begin{abstract}
RESUMEN
El presente trabajo recoge las principales aportaciones de la nueva historiografía sobre el Oriente asiático que ponen en cuestión el supuesto de que las economías europeas tuvieron un desempeño excepcional en los siglos previos a la Revolución Industrial e insisten, en cambio, en el carácter abrupto y tardío de la divergencia en sus trayectorias de desarrollo. Los hallazgos de esa nueva historiografía refuerzan, en primer lugar, la idea de que el dinamismo mercantil es compatible con marcos institucionales muy diversos. Por otro lado, tienden a señalar que en la emergencia del crecimiento económico sostenido concurrieron factores causales diversos, producto de lógicas distintas, que en ningún caso cabe reducir al perfeccionamiento progresivo de las instituciones económicas y el desarrollo gradual de mercados más eficientes.

El artículo concluye con una breve reflexión sobre las implicaciones de ese debate historiográfico para la teorización sobre los fundamentos del desarrollo económico.
\end{abstract}

PALABRAS CLAVE: Desarrollo económico, Comparaciones, Europa, China

Códigos JEL: N01, N20, 010

\section{ABSTRACT}

This article brings together the main contributions of the recent historiography on East Asia which questions the traditional idea about the "exceptionalism" of European economic performance in the centuries prior to the industrial revolution. They suggest instead that divergence in their development paths was a rather late and abrupt phenomenon. The findings of this historiography reinforce, in the first place, the idea that commercial dynamism can occur within very different institutional frameworks. Second, they suggest that the emergence of sustained economic growth was the result of various causal factors which cannot be reduced to the gradual improvement of economic institutions and the development of more efficient markets. The article concludes with a brief reflection on the implications of this historiographical debate for our theorization on the basis of economic development.

KEY WORDS: Economic development, Comparisons, Europe, China

JEL Codes: N01, N20, 010 


\section{Las consecuencias de la desigualdad historiográfica: del eurocen- trismo al determinismo institucionalista ${ }^{1}$}

$\mathrm{N}$ uestras teorías sobre el desarrollo económico se han apoyado tradicionalmente en la reflexión sobre las razones del éxito o del fracaso relativo de las sociedades de Eurasia en el transcurso de la Edad Moderna. Ya en el origen mismo de la ciencia social, Karl Marx y Max Weber ensayaron comparaciones entre Europa y "los demás" para intentar documentar el potencial de progreso material de las configuraciones institucionales presentes en las diversas formaciones históricas euroasiáticas. Ambos, sin embargo, adolecieron de un conocimiento enormemente asimétrico de los mundos que compararon, y aquel desequilibrio perjudicó sin duda a sus teorías sobre las bases institucionales del crecimiento sostenido. Pero Marx y Weber fueron sólo las primeras víctimas de la comparación sesgada, porque lo cierto es que desde entonces la teorización sobre el desarrollo económico ha venido arrastrando constantemente el lastre de "la desigualdad historiográfica entre Europa y el resto del mundo" (O’Brien, 2003, p. 72).

Los europeos fuimos estudiando con detalle nuestro pasado y aislando multitud de rasgos "excepcionales" de nuestra tradición, al parecer ausentes en otras civilizaciones de las que, de entrada, desconocíamos casi todo. Así, creímos descubrir, para lo que nos interesará en este trabajo, que el crecimiento económico intensivo - el crecimiento del producto per capita - fue desde mucho tiempo atrás una característica diferencial de nuestro mundo. Algunos historiadores de la economía terminaron remontando ese fenómeno supuestamente único al momento mismo del "nacimiento de Europa", poco después del año 1000, y describieron cómo fue progresando durante la Edad Moderna mucho antes del inicio de la Revolución Industrial.

Para explicar aquel dinamismo, que ofrecía un contraste tan llamativo con el aparente estancamiento de las otras economías que permanecían en la penumbra, se fue apelando a la superioridad secular de los marcos culturales e institucionales de la actividad económica en Europa. Aquello era perfectamente natural. También lo es que las profundas asimetrías en el conocimiento de la historia hayan estado impulsando desde entonces la construcción de teorías que predican la existencia de una vía única hacia el desarrollo económico, generalizando a partir de algún destilado ideal de la experiencia europea. El evolucionismo clásico en todas sus variedades y las teorías de la modernización de la segunda posguerra, con su sucesión mecánica de estadios, son los ejemplares más conocidos de esta especie de relatos

[Fecha de recepción del original, diciembre de 2005. Versión definitiva, septiembre de 2006]

Deseo agradecer los comentarios a la versión inicial de este artículo a mis compañeros Álvaro Anchuelo y

Gonzalo Ramírez de Haro, y a los informantes de Investigaciones de Historia Económica. 
unilineales $^{2}$. Su mutación más reciente es el hoy triunfante neoinstitucionalismo, asociado a la obra pionera de Douglass North.

No cabe duda de que el magnífico trabajo de North ha contribuido en mucho a corregir el universalismo abstracto de una ciencia económica que en algún momento optó deliberadamente por olvidar la historia ${ }^{3}$. Sin embargo, no es menos cierto que sus propias construcciones teóricas descansan sobre una base historiográfica muy sesgada, dado que en sus narrativas sobre "el nacimiento del mundo occidental" (North y Thomas, 1978) o en sus reflexiones sobre "la paradoja de Occidente" (North, 1993) sólo importan de verdad ciertos datos de la historia europea. Esto no plantearía ningún problema si su intención fuese únicamente ilustrar cómo y por qué el andamiaje institucional en determinadas regiones de Europa occidental fue evolucionando de un modo que promovió el funcionamiento progresivamente más eficiente de los mercados competitivos del ideal neoclásico. Pero North no se limita a eso, por supuesto. Pretende, además, que el desarrollo de ese conjunto preciso de instituciones que impulsaron la competencia en mercados cada vez más abiertos fue el motor del dinamismo económico europeo y, en definitiva, el factor diferencial que explica su divergencia en ingresos respecto al resto del mundo.

De manera que North plantea una doble hipótesis causal: por un lado, se nos dice que la estructura institucional es el principal determinante —en la práctica, el único auténticamente relevante- de las diferencias en el desempeño económico; por otro, se afirma que fue la emergencia de un conjunto superior de instituciones lo que impulsó el "ascenso de Occidente". Pero, al menos si nos atenemos al "método de la diferencia" de Mill, deberíamos esperar, en primer lugar, que se nos mostrara de un modo convincente que tanto el fenómeno a explicar - el crecimiento sostenido - como el explanans — una estructura institucional favorable al desarrollo de mercados competitivos- estuvieron realmente presentes sólo en uno de los casos durante el período que se estudia. En segundo lugar, tendríamos que saber que no existieron entre los casos otras diferencias distintas de la propuesta, y tal vez tanto o más relevantes, para dar cuenta de las variaciones en los resultados (Little, 1991, p. 36). Sin embargo, no obtenemos nada de esto, dado que, "desigualdad historiográfica" mediante, en la historia que se nos relata las trayectorias de las otras civilizaciones están ausentes o reducidas a una caricatura sucinta. De

2 Véase Sztompka (1995) para una buena exposición de las distintas versiones del evolucionismo y las teorías de la modernización.

3 Hodgson (2001). Según Robert Solow, la economía, como disciplina científica, aspira a convertirse en la "física de la sociedad", dotada de "un único modelo del mundo universalmente válido". Citado en North (2005), p. 19. 
modo que North nos cuenta cómo arraigaron en Europa determinadas instituciones que respaldan a los mercados y que la teoría neoclásica da por supuestas sin más. Pero él a su vez da por supuesto que es el desarrollo de ese conjunto preciso de "buenas instituciones" lo que da cuenta del "milagro" del crecimiento sostenido. Lo que resulta entonces es una generalización a partir de un único caso y por ello otro universalismo infundado.

Las consecuencias políticas de los relatos unilaterales sobre el "milagro europeo" son suficientemente conocidas. Obligan siempre a concluir que el desarrollo económico ocurre a través de la difusión de los modelos culturales e institucionales de Occidente y que su extensión al resto del mundo sólo podrá proceder de una "globalización imitativa" de una fórmula europea original (Chakrabarty, 2000, p. 7). En los últimos años esto se ha estado traduciendo en la práctica del "monocultivo institucional": la pretensión, en ocasiones muy agresiva, de implantar en todas partes un conjunto preciso de instituciones, destilado de alguna interpretación idealizada de la experiencia histórica de Occidente y en nuestros días con un clarísimo sesgo anglosajón (Evans, 2004). En definitiva, la exportación del andamiaje institucional que se supone indiscutiblemente superior, porque esa historia así lo "demuestra", para impulsar el desarrollo económico.

Las prácticas del "monocultivo institucional" podrían discutirse desde un punto de vista normativo si se piensa, por ejemplo, que el carácter determinista del saber experto que las sostiene arrasa la diversidad cultural y ahoga la deliberación democrática ${ }^{4}$. Sin embargo, si el experto tuviese razón al proponer un óptimo institucional como motor principal y casi único del crecimiento económico, siempre podría refugiarse en la distinción weberiana entre ciencia y política, y afirmar, con David Landes, que el paquete ideal de instituciones que propone la historia del ascenso de Occidente no describe "una sociedad "mejor" ni "superior", sino simplemente una sociedad mejor preparada para producir bienes y servicios» (Landes, 1999, p. 206). Nada que objetar entonces si algunos humanos por cualquier razón decidimos dar la espalda al crecimiento económico para perseguir otros fines colectivos, porque la ciencia no enseña a nadie «qué debe hacer, sino únicamente qué puede hacer» para conseguir aquello que desea (Lechner, 1986, p. 26). Ahora bien, en el caso de que deseemos el crecimiento, la historia del "milagro europeo" muestra claramente lo que hay que hacer para obtenerlo. Y si esto fuera así de sencillo, en materia de políticas para promover el crecimiento económico la cuestión se reduciría a la postre, como asegura Landes con su mordacidad y contundencia

4 Es en la antropología del desarrollo donde se han elaborado las críticas más radicales del "saber experto" de los economistas como discurso de dominación que niega de raíz el derecho a la diferencia. Pueden verse, por ejemplo, Escobar (1997) y Ferguson (1994). 
características, a una simple elección entre «la verdad y lo políticamente correcto» (Landes, 1999, p. 18). Pero ocurre que no lo es. Más bien lo único que parece una "verdad" indiscutible en este tiempo es que debería resultar ya imposible mantener con tanto aplomo ese estilo de disyuntiva dogmática.

Una manera de comenzar a disolver las certidumbres deterministas es desde luego describir en toda su complejidad la realidad del crecimiento económico en el presente. Parece difícil, en efecto, no sacar conclusiones del hecho de que durante las últimas décadas la prosperidad se haya ido extendiendo a un grupo bastante significativo de sociedades no occidentales con marcos institucionales muy distintos, mientras el desempeño de los países que adoptaban reglas institucionales ortodoxas y estándares canónicos de buen gobierno era a su vez enormemente heterogéneo (Rodrik, 2003, p. 4).

En este trabajo ensayaré un camino alternativo, argumentando que los nuevos fragmentos que la historiografía "poscolonial" ha estado incorporando al calidoscopio de la historia nos ofrecen una imagen de la realidad diversa del crecimiento económico en el pasado que también encaja muy mal con las certezas de aquella "verdad" convencional. En concreto, los detalles que van emergiendo de esos estudios cuestionan radicalmente nuestros supuestos más consolidados sobre el carácter excepcional del crecimiento económico europeo en los siglos que precedieron a la Revolución Industrial. Revelan, en cambio, un mundo de "semejanzas sorprendentes" entre las sociedades más avanzadas de los dos extremos de Eurasia. Y esto no es en absoluto trivial si pensamos en que el relato dominante sobre los fundamentos institucionales de la prosperidad de las sociedades se ha construido precisamente sobre la base de la supuesta excepcionalidad secular de la experiencia europea.

Como el progreso de la ciencia social depende críticamente del estudio comparado de la historia, debería resultar evidente que, si deseamos comprender mejor los determinantes de la riqueza y la pobreza de las naciones, haríamos bien en interesarnos más por el pasado de los otros pueblos y tomarnos realmente en serio los hallazgos de quienes se dedican a estudiarlo. Es posible que el estudio detallado de las semejanzas y las diferencias entre las instituciones y los resultados de las distintas regiones de Eurasia nos impulse a reevaluar nuestras atribuciones de centralidad causal a ciertas variables y procesos, y tal vez a atribuir un nuevo énfasis a otras. Y puede que, si nos esforzamos por incorporar a nuestras reflexiones la riqueza de los relatos que van saliendo de la sombra para corregir lentamente la secular desigualdad historiográfica entre Europa y el resto del mundo, consigamos dotar de un mayor realismo a nuestras teorías sobre las bases del desarrollo económico. 


\section{El excepcionalismo europeo cuestionado: mercados y desarrollo económico en Oriente y Occidente}

El esfuerzo por datar con un cierto fundamento el origen de la divergencia entre los niveles de vida de las poblaciones de las grandes culturas mercantiles en los dos extremos de Eurasia - la pregunta empírica por el "cuándo" - debería ser un paso previo para cualquier intento de iluminar las causas de la brecha en su riqueza relativa - la pregunta teórica sobre el "por qué" - Sin embargo, desde que los estudiosos de la historia económica de Europa dinamitaron definitivamente nuestra imagen de las sociedades preindustriales como mundos inmóviles, al demostrar la presencia de varios elementos del crecimiento económico moderno - un crecimiento macroeconómico sustancial acompañado por cambios técnicos y aumentos en el ingreso per capita- mucho antes del año $1800^{5}$, lo habitual ha sido dar sencillamente por supuesta la existencia de una ancestral "excepcionalidad" europea en materia de dinamismo económico ${ }^{6}$.

China ha sido tradicionalmente el contrapunto ideal para el relato gradualista sobre el ascenso secular del núcleo dinámico europeo. Una civilización que mantuvo un prolongado liderazgo tecnológico; que probablemente en algún momento casi con seguridad entre los siglos X y XIII — dio también muestras de un "auténtico crecimiento económico"; pero que se estancó poco después, tal vez presa de un despótico "estado centralizado" que actuaba "al albur de los caprichos de un emperador", mientras las regiones más avanzadas de Europa continuaban progresando en la ruta hacia el crecimiento sostenido (North, 1993, p. 9).

La investigación histórica más reciente está esbozando, sin embargo, una imagen muy distinta de la trayectoria de la economía china en la Edad Moderna. Parece, en efecto, que no sólo no se mantuvo estancada en un sopor secular mientras la ágil Europa progresaba en su asalto a la prosperidad, sino que conoció un espectacular florecimiento económico como mínimo entre 1680 y 1780, durante el período central de la dinastía Qing (Manchú). Un largo episodio de crecimiento que permi-

5 Holanda, el caso mejor documentado de expansión económica sostenida en el período previo a la Revolución Industrial, recibió en su momento el grado honorífico de "primera economía moderna» (De Vries y der Woude, 1997). Pero también cabe encontrar episodios sustanciales de crecimiento en Europa poco después del año 1000. De modo que tampoco carecería de sentido remontar hasta entonces el origen de nuestra "modernidad temprana", como propone Levine (2000).

6 Según David Landes, todas las grandes civilizaciones "se estaban quedando rezagadas con respecto a Europa" ya en pleno corazón de la Edad Media (Landes, 1999, p. 63). Puede que Landes sea particularmente extremista en su afirmación de la superioridad milenaria de la civilización europea, pero no está ni mucho menos solo, y conviene recordar en cualquier caso que probablemente se vendieron más copias de su libro en los dos años que siguieron a su publicación en 1998 que de todos los demás textos de historia económica juntos (Vries, 2001, p. 33). 
tió que la población se doblase con creces en el siglo XVIII — de 160 millones en 1700 a 350 en 1800-, mientras el nivel de vida también mejoraba ${ }^{7}$. De modo que en el siglo XVIII la esperanza de vida en las zonas más prósperas de Europa y China era aproximadamente la misma, en tanto que el consumo de calorías por adulto y día en el conjunto de China era equivalente a la cantidad estimada para Inglaterra a mediados del XIX (Pomeranz, 2000, pp. 36-43).

China es - junto a Japón- el área geográfica donde los indicios que apuntan a la existencia de un nivel de prosperidad general equivalente al de Europa occidental en vísperas de la Revolución Industrial son más abundantes y creíbles, aunque los progresos de la historiografía "poscolonial" tienden a ir presentando imágenes sorprendentes del bienestar material también en otras zonas de Asia ${ }^{8}$. Pero no es necesario comulgar con cada uno de los datos que nos ofrecen los nuevos historiadores del Oriente, ni compartir por completo el entusiasmo de algunos autores con el "milagro asiático" de la Edad Moderna 9 , para aceptar que los nuevos fragmentos que han ido introduciendo en el calidoscopio de la historia económica hacen muy difícil continuar defendiendo sin más matices el relato gradualista dominante.

Eric Jones, autor del célebre El milagro europeo, ha reconocido en su obra más reciente que los nuevos hallazgos historiográficos hacen necesario reevaluar en profundidad la evolución histórica de la productividad en la economía china (Jones, 2002, p. 44). No cree, sin embargo, que el descubrimiento de aquellas "semejanzas sorprendentes" entre los niveles de vida en Europa y el Oriente asiático en fecha tan tardía nos obligue también a revisar la idea de que "la industrialización fue el resultado del crecimiento intensivo" de las economías europeas en el transcurso de los siglos previos, y en ningún caso la causa de su divergencia en ingresos con el resto del mundo (Jones, 2002, p. 4). Podría estar en lo cierto, pero para convencernos debería demostrar que existió alguna diferencia crítica en la dinámica económica de los dos extremos de Eurasia que hiciese previsible el estancamiento de los ingresos en un caso y el progreso hacia la industrialización y el crecimiento sostenido en el otro.

En un artículo magistral publicado hace ya una década, Jan de Vries (1994) acunó la expresión "revolución industriosa" para describir lo que percibía como la esencia de la transformación económica que tuvo lugar en las áreas más comercializadas de Europa occidental durante los siglos que precedieron a la Revolución

Las cifras de población son de Lee y Feng (1999), p. 27.

Por ejemplo, Parthasarathi (2001) ha estimado recientemente que los ingresos de los tejedores en el sur de la India eran superiores en términos reales a los de los tejedores británicos a mediados del siglo XVIII. Citado en Bayly (2004), p. 57.

9 Seguramente fue André Gunder Frank quien, en sus últimos trabajos, llegó más lejos en el empeño por demostrar la superioridad de las grandes economías del Oriente durante toda la Edad Moderna. Véase sobre todo Frank (1998). 
Industrial. Su idea era que la fuerza motriz del crecimiento económico durante aquella larga etapa fue un aumento del esfuerzo de trabajo motivado por el deseo de conseguir más bienes de consumo sólo accesibles en el mercado. Una buena parte de éstos procedía de ultramar - algunos eran "alimentos adictivos", como el azúcar, el tabaco, el cacao o el café- y los hogares podían seguir diversas estrategias para obtenerlos: especializarse en el cultivo de determinados productos agrícolas, dedicarse a la producción protoindustrial $\mathrm{u}$ ofertar trabajo asalariado o servicios comerciales (De Vries, 2003, pp. 47-48).

Si aceptamos que en aquel proceso se incrementó la carga de trabajo y la propensión de los hogares a enviar a trabajar para el mercado a mujeres y niños, podemos entender por qué pudieron caer los salarios reales (por hora trabajada) en el siglo XVIII y en las primeras décadas del siglo XIX, mientras aumentaba simultáneamente el consumo. También, y para lo que ahora nos importa, si estos desarrollos fuesen en verdad privativos de determinadas regiones de Europa podríamos descartar como irrelevantes los datos sobre esperanza de vida o ingesta de calorías en las áreas más prósperas del Oriente en vísperas de la revolución industrial -la "cliometría calorimétrica" que Landes (1999, p. 29) menciona con su habitual displicencia- Habríamos identificado una diferencia específica en el crecimiento económico europeo, un dinamismo único cuya prueba no debe buscarse en el aumento general del nivel de vida, sino en el consumo creciente de "lujos" que sólo podían adquirirse en el mercado y que movilizaba por ello el esfuerzo productivo, impulsando la especialización y el aumento de la productividad.

Ocurre, sin embargo, que los hallazgos de la nueva historiografía parecen revelar que el consumo de "lujos" estaba también muy extendido entre los hogares de las regiones más prósperas del Oriente (Pomeranz, 2000, pp. 116-152). Los datos, por supuesto, permanecen en disputa ${ }^{10}$. Pero precisamente porque los datos no obligan, la justificación teórica de las diferencias que se postulan debería ser especialmente convincente. Jan de Vries da cuenta de los cambios en el comportamiento económico de los hogares que desencadenaron la dinámica del crecimiento "smithiano" en Europa, y no en China, sin apelar a variaciones en las preferencias de los individuos derivadas de alguna transformación cultural, que plantean siempre dudas irresolubles sobre la dirección de la causalidad. Su explicación teórica descansa exclusivamente sobre las variables del entorno de elección del homo œconomicus. Lo que tenemos, en primer lugar, son nuevas oportunidades de consumo y, a continuación, la idea de que el proceso sólo pudo desencadenarse y avanzar realmente en aquellas sociedades en que los hogares tenían la oportunidad de decidir libremente a qué dedicar sus

10 De entrada, porque no disponemos para China de nada equivalente a los inventarios que acompañaban a los testamentos en Europa, que son la principal fuente de información de De Vries. 
energías y cierta seguridad de recibir los beneficios de su esfuerzo. Es ciertamente el modo de mirar habitual del economista: las motivaciones individuales son una constante universal, de modo que si prevalecen determinados estándares institucionales que garanticen el acceso igualitario y seguro a los mercados, "el 'amor al lucro' hará el resto" (Skidelsky, 2001, p. 28).

Pero la historiografía revisionista continúa poniendo piedras en el camino del relato gradualista, dado que ha estado intentando mostrar también que durante aquel mismo período había en China tanta libertad de mercado, cuanto menos, como en las regiones más avanzadas de Europa occidental. Según la descripción de Ken Pomeranz, en China el mercado para la tierra era libre, aunque parcialmente limitado por la costumbre - los familiares tenían una opción de compra preferente-, y el porcentaje de la tierra inmovilizada era además insignificante en comparación con las situaciones que prevalecían por entonces en Europa. En cuanto al mercado de trabajo, la servidumbre fue desapareciendo al mismo ritmo en ambas regiones y parece que las familias campesinas chinas tenían una mayor libertad tanto para elegir a quién vender sus productos como para participar en la producción artesanal destinada al mercado (Pomeranz, 2000, pp. 69-107). Si otorgamos alguna credibilidad a estas descripciones, deberíamos concluir que en China los mercados no estaban más alejados del ideal neoclásico que en las regiones más avanzadas de Europa occidental, e incluso probablemente estaban más cerca. Y si esto es así, ¿cómo explicamos entonces el hecho de que las economías europeas continuasen y profundizasen su crecimiento inducido por el mercado mientras la economía china se estancaba desde finales del siglo XVIII para precipitarse después en una espiral regresiva?

El propio Jan de Vries, en un manuscrito inédito en el que intenta responder al desafío de la nueva historiografía sobre el Oriente, se limita a constatar su "impresión" de que las decisiones de los hogares asiáticos reflejan un contacto en ocasiones intenso con el mercado - lo que explicaría su mayor laboriosidad y el aumento en el consumo de "lujos"-, pero no una orientación hacia el mercado. Esta ausencia de una orientación hacia el mercado entre los hogares chinos debió abortar el progreso de la especialización y los incrementos de la productividad que se derivan de ella, y que sentaron en Europa "las bases para la revolución industrial, un acontecimiento que continuó más que inició el proceso de desarrollo económico"11 . Lo que no sabemos, sin embargo, es por qué ocurrió aquello: por qué los hogares europeos "optaron" por depender más estrechamente del mercado que los chinos. La historia falla, en definitiva, porque no es capaz de hacer inteligible el hecho de que los "industriosos" hogares chinos, que disponían de una tecnología cuando menos equivalente a

11 De Vries (2001), p. 9. Las cursivas son del original. Agradezco a Jan de Vries su permiso para citar este trabajo. 
la europea, que emplearon estrategias alternativas para alcanzar niveles de fertilidad semejantes a los europeos ${ }^{12}$ y que disfrutaban de una amplia libertad para vender y comprar en el mercado, no siguieran a la postre el mismo camino que los europeos.

La sociología histórica de raigambre marxista defiende un relato mucho menos amable de los orígenes del crecimiento "smithiano" que, sin embargo, sí permite entender el porqué de las diferencias en el grado de mercantilización de las economías preindustriales. Para los autores de esta tradición la clave del cambio estuvo, como sabemos, en la emergencia de unas relaciones de propiedad "capitalistas" que aparecieron como resultado históricamente contingente de los conflictos de clase. Tal como defendió Marx en su momento, fue la destrucción violenta de la economía tradicional inglesa lo que terminó obligando a los agentes económicos a atenerse a la lógica del mercado (Brenner, 1989). Para hacer inteligible esta historia no es necesario detenerse a intentar aclarar las razones por las que en unas regiones y no en otras los hogares optaron por vincularse al mercado en lugar de limitarse a mantener "contactos" con él. Las decisiones autónomas de los hogares resultan irrelevantes porque en un lugar determinado desapareció la base tradicional de su supervivencia, dejándoles sencillamente sin opciones. El gigantesco proceso de redistribución de la tierra que ocurrió en Inglaterra, mediado por el conflicto sociopolítico y las coacciones institucionales, les obligó a depender del mercado para ganarse la vida, bien como empresarios agrícolas, bien como asalariados. La lucha de clases, y no el desarrollo gradual y pacífico de mercados más amplios y abiertos, es aquí el motor de la historia y lo que explica los cambios en el comportamiento de los agentes económicos. Se trata de una narración clara y precisa y que tiene la ventaja adicional de centrar el argumento en Inglaterra, la región de Europa occidental donde la tierra y el trabajo llegaron a estar más estrictamente separados y más completamente mercantilizados... y donde finalmente arrancó la Revolución Industrial.

12 En otro tiempo podría haberse argumentado que, por mucho que el marco institucional en su conjunto fuese favorable al desarrollo del mercado, los orientales, al contrario de los europeos, no fueron capaces de mantener el crecimiento demográfico por debajo del máximo, y que esto evitó que el crecimiento del consumo desencadenase allí la espiral virtuosa. Pero las investigaciones más recientes sobre la historia demográfica en China han restado cualquier credibilidad a la famosa tesis de Hajnal, según la cual los hogares europeos, y sólo ellos, se las ingeniaron para mantener el crecimiento de la población por debajo de su nivel máximo, evitando, en palabras de H. G. Wells, “agotar los recursos de su entorno con una multiplicación insensata de la vida" (Jones, 1991, pp. 57-66). Parece que al llegar a las variaciones en el comportamiento reproductivo inducidas por las distintas instituciones familiares, nos encontramos de nuevo con un caso de comparación asimétrica en el que la hipótesis causal va perdiendo credibilidad a medida que vamos avanzando en el conocimiento del término que estaba ausente. Hoy sabemos, en efecto, que la planificación familiar no fue nunca un monopolio de Europa, y que cuando menos en China "el control de la fertilidad estuvo siempre en la esfera de la elección consciente y calculada" (Lee y Feng, 1999, p. 21). 
Desde luego, el marco institucional de la Inglaterra capitalista, con su nueva estructura de derechos de propiedad que dotaba de una nueva seguridad a unos erosionando la posición de muchos otros, devino muy distinta a la de la economía mercantil china, y ello tuvo sin duda consecuencias para sus patrones de desarrollo económico. Parece probado, por ejemplo, que la redistribución de la propiedad y el impulso simultáneo hacia una mayor mercantilización de la economía terminaron produciendo un incremento notable de la productividad del trabajo en la agricultura inglesa, de modo que ésta, que partía de un nivel bastante inferior a comienzos del siglo XVIII, era tal vez un 20 por 100 superior a la del delta del Yangtzé -la región más próspera de China- en $1800^{13}$. Aquel progreso sustancial de la productividad del trabajo en la agricultura pudo muy bien ser una peculiaridad inglesa, que cabría explicar a partir del impulso sistemático a reducir los costes en una economía que estaba sufriendo una mercantilización particularmente intensa. Pero conviene reflexionar un momento sobre el significado de este proceso y el papel que solemos atribuirle en la emergencia de una dinámica de crecimiento económico sostenido.

Es fácil ver que el incremento en la productividad del trabajo en el sector agrícola no implica necesariamente ni un aumento del ingreso agregado, ni una mejora en la capacidad de la agricultura como un todo para sostener al conjunto de la población. Si tienen lugar cambios sustanciales en la distribución de la tierra y en la proporción de la población trabajando en el campo, la productividad del trabajo puede crecer de un modo espectacular sin que aumente la producción total y sin que el conjunto de la economía se beneficie realmente del cambio, a no ser que la población desplazada encuentre trabajo en ocupaciones más productivas (Goldstone, 2002a, pp. 22-23). En el caso de la "revolución agrícola" inglesa, los rendimientos globales y por hectárea se mantuvieron al parecer constantes durante el siglo XVIII. Es más: según relata Mauro Ambrosoli, cuando los ingleses estudiaron las prácticas agrícolas continentales, los manuales clásicos y los resultados de su propia experiencia, descubrieron que la mayor parte de lo que podía aprenderse sobre las técnicas para mantener y mejorar los rendimientos de la tierra no se ponía en práctica en Inglaterra, sencillamente porque implicaba métodos muy intensivos en trabajo que chocaban frontalmente con las prácticas de los granjeros capitalistas, empeñados en la reducción de los costes laborales y la maximización de los beneficios ${ }^{14}$. Siendo esto así, tiene pleno sentido preguntarse si aquella "revolución agrícola" capitalista era necesariamente, como nos hemos acostumbrado a pensar, una transformación virtuosa en el camino de ascenso hacia el crecimiento sostenido o más bien, por el contrario, el arranque de una trayectoria suicida.

13 Goldstone (2002a), p. 22. Puede verse también un repaso detallado de la evidencia en Allen (2003).

$14 \quad$ Ambrosoli (1997). Citado en Pomeranz (2000), p. 216. 
Recitar las características peculiares del andamiaje institucional en Inglaterra o en China es algo muy distinto que demostrar que ambas regiones estaban claramente embarcadas durante el período preindustrial en trayectorias divergentes. El rendimiento global de las economías era muy parecido y tampoco los contrastes más visibles en las consecuencias distributivas de los distintos marcos institucionales daban a priori una ventaja evidente a alguna de las partes. Sabemos, por ejemplo, que, aunque en la antesala de la Revolución Industrial los ingresos de los hogares en el delta del Yangtzé e Inglaterra eran aproximadamente los mismos y la distribución del tiempo de trabajo entre faenas agrícolas e industriales muy semejante, el desplazamiento del trabajo de la agricultura hacia la manufactura había seguido unas pautas muy diferentes (Allen, 2003, p. 15). En Inglaterra, separando de las labores agrícolas a un porcentaje creciente de los hogares; en China, apartando a las mujeres del trabajo en los campos e integrándolas en la industria doméstica (Goldstone, 2002a, p. 31). El argumento marxista es que la mayor disposición de los ingleses a desplazarse y trabajar por un salario no fue el resultado de la racionalidad económica de los hogares en su interacción con el mercado, sino de las consecuencias distributivas del marco institucional que resultó de unos conflictos sociopolíticos que favorecieron a determinados grupos e incrementaron la vulnerabilidad e inseguridad del campesinado. Pero para lo que aquí nos interesa lo importante es que sólo sabiendo lo que vino después cabría argumentar que la vía "capitalista" inglesa - perversa desde el punto de vista del hogar campesino y probablemente irrelevante para la eficiencia global de la economía - tenía en cualquier caso la clara ventaja de liberar más mano de obra para las fábricas del futuro.

De modo que si queremos tomarnos en serio la historia haríamos bien en abandonar nuestra atalaya privilegiada para pensar que a priori nada garantizaba que hubiese un final feliz en cualquiera de los polos. Por el momento, los mercados en China e Inglaterra, con sus marcos institucionales diversos, estaban produciendo resultados muy semejantes en términos de productividad e ingreso agregado, y seguramente se enfrentaban a problemas muy parecidos para sostener el crecimiento económico en el futuro. No estaba escrito en ninguna parte, por ejemplo, que Inglaterra no se viese obligada finalmente a devolver a la tierra a una parte cada vez mayor de los expulsados por la "revolución agrícola" para alimentar a su población en aumento, aplicando técnicas intensivas en trabajo para incrementar la productividad del suelo y conservar su fertilidad, y alejándose así del camino que finalmente condujo a la Revolución Industrial. 


\section{3. "Manos visibles" y relaciones de poder: una nueva visita al colo- nialismo}

El que la divergencia entre Europa y el resto del mundo deberá explicarse siempre, con independencia de cuándo la situemos en el tiempo, haciendo referencia a alguna peculiaridad de las "instituciones" europeas, es casi una tautología ${ }^{15}$. También la vieja narrativa que sitúa la conquista, el saqueo y la dominación de otros pueblos en el corazón del desarrollo económico europeo, el rival más conspicuo del relato gradualista dominante centrado en el perfeccionamiento progresivo de los mercados, tiene un fuerte componente institucional. Si no lo tuviera, su historia sería estrictamente arbitraria. James Blaut quería que lo fuera, empeñado como estaba en mantener hasta el final que "Europa no tenía nada de peculiar" y que todo lo ocurrido empezó por "una cuestión de ventaja en la localización", de tal modo que, si los tesoros de América hubiesen estado en otra parte, posiblemente "la India se hubiese convertido en el hogar del capitalismo y el dominador del mundo" (Blaut, 1993, p. 181). Por supuesto, la geografía, el principal rival de las instituciones entre los determinantes "profundos" de la fortuna de las naciones, importa siempre en todas las historias sobre el desarrollo de la economía mundial ${ }^{16}$. Pero si no nos conformamos con ese estilo de "explicación" azarosa, el intento de comprender tanto la dinámica de los "descubrimientos", la conquista y la colonización, como sus efectos sobre las economías, nos obligará a incluir elementos institucionales que, más allá de su localización y características geográficas, sí debieron hacer de Europa un territorio "peculiar" en algún sentido.

Sabemos que lo habitual, cuando se trata de hacer inteligibles las fuentes del dinamismo colonizador de Europa, es referirse a su fragmentación política, señalando cómo los continuos conflictos bélicos en el seno del sistema de estados proporcionaron

15 Cuando menos si definimos las "instituciones" de un modo suficientemente amplio, capaz de incluir las reglas informales o las variables que en ocasiones se agrupan en el cajón de sastre de la "cultura". ¿De qué otra cosa podría tratarse? Sin duda Taylor (1998) tiene razón cuando recuerda que si la "nueva historia económica" nos ha enseñado algo que deberíamos retener es que, más allá de los modelos neoclásicos, las instituciones hacen una diferencia crítica en los resultados económicos. La pierde, sin embargo, cuando supone que las instituciones están presentes sólo en las historias centradas en las fuentes internas del dinamismo económico europeo, y no en "las desacreditadas teorías estructuralistas/dependentistas de los años 60" que resaltaban sus fuentes externas, apuntando a las relaciones entre Europa y otras partes del mundo. Dudar de que el "ascenso de Occidente" pueda explicarse casi exclusivamente a partir de la calidad de las instituciones que fueron apuntalando el funcionamiento de los mercados competitivos en Europa no equivale en absoluto a contar una historia en la que las instituciones desempeñen un papel secundario.

16 La referencia ineludible cuando se va a parar al influjo de las variables geográficas sobre los procesos de desarrollo económico en el largo plazo es Diamond (1998). En los últimos años son ya muchas las contribuciones a una discusión que ha ido adoptando la forma de una suerte de combate econométrico "geografía versus instituciones". 
el incentivo sistemático para las innovaciones en tecnología militar, o cómo impulsaron su incesante expansión ultramarina en una suerte de extensión hacia el mundo entero de la competencia interestatal europea. A continuación, en todos los relatos de la "acumulación originaria" también ocupan un lugar central la capacidad y disposición de los estados para proteger la economía local y el ejercicio de su poder militar para conquistar territorios, someter a la población indígena o desplazar a los competidores de las regiones y las rutas comerciales más atractivas. Es fácil ver entonces que lo que se discute no es si, en general, "las instituciones importan", porque éstas siempre terminan ocupando un lugar central en cualquier relato creíble sobre el "ascenso de Occidente". De lo que se trata es de qué elementos institucionales selecciona y resalta cada narración cuando viene a interpretar la historia del desarrollo económico. Y, en general, quienes enfatizan la importancia de las relaciones de intercambio asimétrico que Europa estableció con el resto del mundo durante la Edad Moderna, destacan el papel clave que desempeñaron instituciones distintas de las del mercado y que en gran medida actuaron sistemáticamente contra la lógica del mercado libre.

La sórdida historia de la colonización ocupa a lo sumo un lugar marginal en los relatos dominantes sobre las bases institucionales del "milagro europeo", que tienden a interpretar aquellos acontecimientos de un modo que no afecta a la lección esencial de la historia, que versa, invariablemente, sobre las bendiciones de la libertad de mercado. Desde luego, la idea de que sólo los estados europeos que disponían de unas "buenas instituciones" aprovecharon realmente las nuevas oportunidades abiertas por el comercio atlántico, se lleva muy bien con los datos que conocemos de la historia económica europea ${ }^{17}$. Pero si decidimos mirar más allá e incorporar el otro extremo de Eurasia al universo de casos, nos gustaría saber también si, y en su caso por qué, la expansión ultramarina contribuyó a dotar al conjunto de Europa de un mayor potencial de crecimiento frente a otras regiones. Al parecer China tuvo una economía mercantil muy dinámica, pero ciertamente no tuvo colonias, y si esta diferencia fuese importante la historia de la expansión ultramari-

17 Daron Acemoglu y asociados han intentado demostrar que el diferencial de crecimiento en la Europa moderna respecto al período medieval se debió en su totalidad a la aportación de los estados con acceso a la fachada atlántica. A partir de ahí, su argumento es que las sociedades de la fachada atlántica europea en las que existía mayor libertad de entrada al mercado, aquéllas con instituciones políticas menos "absolutistas", en las que los derechos de propiedad estaban más extendidos y mejor protegidos, aprovecharon mejor de entrada las nuevas oportunidades que ofrecía la ampliación de las rutas comerciales, y que la expansión del comercio fortaleció a su vez a los actores más interesados en limitar el poder arbitrario de los gobernantes y consolidar las instituciones del mercado libre. Seguramente esta interacción que proponen entre geografía — el tener o no acceso a la fachada atlántica- e instituciones puede explicar bastante bien las diferencias en el crecimiento dentro de Europa en los siglos previos a la revolución industrial. Véase Acemoglu, Johnson y Robinson (2005). 
na podría tal vez enseñarnos algo distinto de lo que hemos ido aprendiendo al comparar la trayectoria de las diversas sociedades dentro de una región que sí las tuvo.

En una primera aproximación, podría parecer que el descubrimiento del dinamismo económico de la China moderna tiende más bien a confirmar la hipótesis, ampliamente compartida por los historiadores de la economía, de que la contribución de la aventura colonial al crecimiento fue, de haber existido, insignificante en comparación con la acumulación de recursos en la miríada de intercambios impulsados por el desarrollo del mercado dentro de Europa. Si China vivió un florecimiento comparable al de los casos europeos más exitosos, si gozó de una prosperidad equivalente sin colonias, tendríamos en principio una razón adicional para dudar del valor de aquéllas. Una segunda lectura, sin embargo, podría llevarnos a pensar lo contrario. Porque cabría muy bien sospechar que si el dinamismo económico inducido por el mercado fue realmente tan semejante en los dos extremos de Eurasia, tal vez el colonialismo desempeñase al fin un papel determinante para decidir la suerte que correría a la postre cada uno. Un papel más importante de lo que podemos intuir si nos limitamos a comparar las distintas trayectorias dentro de Europa. Un papel más decisivo de lo que nos fuimos acostumbrando a creer mientras supusimos que las economías mercantiles europeas eran, desde tiempo atrás, mucho más dinámicas que la china y que la conquista, por lo tanto, fue un reflejo de esa superioridad, pero en ningún caso contribuyó a crearla.

Por otro lado, los argumentos de quienes piensan que el colonialismo ocupó un lugar central en el "ascenso de Occidente" nunca se han limitado a recordarnos, en la vena de Williams, los "beneficios extraordinarios" que los europeos obtuvieron mediante el saqueo y la coacción. Las hipótesis de Ken Pomeranz apuntan, por ejemplo, en una dirección muy diferente. Para él la clave del proceso estuvo en la creación de un "nuevo tipo de periferia" que permitió sostener la especialización industrial de determinados lugares de Europa, en particular de Gran Bretaña, más allá de lo que hubiese sido posible de haber prevalecido un comercio más consensual, más libre, más parecido, en definitiva, al que tuvo lugar entre las diversas economías regionales en el Extremo Oriente. Su idea es que en el mundo preindustrial, cuando no existían tecnologías costosas y complejas ni grandes brechas en la productividad, el intercambio libre entre las regiones más avanzadas y más atrasadas conducía de un modo casi "natural" a la industrialización por "sustitución de importaciones", a medida que aumentaba la población en las regiones más pobres y la explotación de tierras más y más alejadas de las costas y las vías fluviales hacía caer los rendimientos de la exportación de alimentos y materias primas. La conquista y colonización de América, sin embargo, permitió a Europa trascender los límites sistemáticos a la especialización regional que, con la excepción de algunas manufacturas que exigían conocimientos muy difíciles de adquirir, operaron en cambio de un modo continuo en el comercio chino a larga distancia. 
En particular, la "gran mortandad" y la repoblación con mano de obra esclava convirtieron a la región en torno al Caribe en el socio comercial "ideal" para una región industrial naciente. Lo fundamental, desde el punto de vista de Pomeranz, no es el tamaño del mercado colonial sino el hecho de que el Nuevo Mundo y el tráfico de esclavos ofrecían algo que ningún otro mercado podía ofrecer: un área social y políticamente "configurada" para mantener un intercambio continuo de bienes de capital —en este caso, en forma de gente secuestrada - y manufacturas de consumo, por unas cantidades crecientes de bienes intensivos en tierra - alimentos, fibras y más tarde madera- a precios razonables ${ }^{18}$.

Es evidente que el énfasis en los beneficios que se derivaron del proceso político de la colonización, de la construcción coactiva de una economía mundial diferenciada y asimétrica, resultará más creíble cuanto más dispuestos estemos a aceptar que no existían diferencias excesivamente importantes en otros ámbitos entre las sociedades más avanzadas de los dos extremos de Eurasia. Si fuese cierto que el dinamismo de los mercados era semejante, aunque sus marcos institucionales fuesen distintos, y que todas las economías orgánicas más mercantilizadas e industrializadas enfrentaban un problema de escasez de tierra que no podía solucionarse mediante el comercio libre, consensual, tendría sentido pensar que las características peculiares de la economía colonial pudieron hacer realmente una diferencia crítica en sus trayectorias de desarrollo.

Podríamos imaginar, por ejemplo, que Inglaterra hubiese sido incapaz de mantener su "modelo" de desarrollo capitalista en la agricultura de no haber dispuesto del flujo constante de recursos intensivos en tierra que le proporcionó el triángulo atlántico. Tal vez hubiese tenido que embarcarse, como sugerí más arriba, en una ruta de "recampesinización", devolviendo a una parte de su población a la tierra para mejorar sus rendimientos y sostener el crecimiento demográfico, acercándose al "modelo de desarrollo" chino o danés - a las economías mercantiles que dependían en mucha mayor medida de la explotación intensiva de su propia tierra- y abandonando el camino que condujo a la Revolución Industrial. Después de todo, vimos que la "revolución agrícola" inglesa apenas había mejorado la productividad de la tierra; de hecho, parece que la relación entre población y producto agrario estaba empeorando en la segunda mitad del siglo XVIII.

18 Pomeranz (2000), pp. 264-74. Por otro lado, los metales preciosos obtenidos en la América hispana mediante el empleo de otras instituciones "peculiares" de trabajo forzoso lubricaron los flujos comerciales y permitieron a las regiones más avanzadas de Europa occidental obtener los "recursos reales" que necesitaban para proseguir con su especialización industrial más allá de lo que hubiera sido posible sin ellos. La plata americana sostuvo el comercio con áreas ecológicamente ricas en las que los mercados para los productos manufacturados europeos eran reducidos o inexistentes, como Europa oriental y el Sudeste asiático; indirectamente, contribuyó al crecimiento de las economías de plantación en el Caribe al pagar los textiles indios que se cambiaban por esclavos en las costas de África. 
Por supuesto, no está en absoluto claro que los recursos de ultramar fuesen realmente necesarios, como pretende Pomeranz, para la continuidad del crecimiento en las regiones más avanzadas de Europa. Es posible, por ejemplo, que las comparaciones más recientes sobre productividad agraria en Inglaterra y China tiendan a subestimar la capacidad del campo inglés para mantener a su población. Se trata de comparaciones centradas en los granos de mayor calidad, y sabemos que los ingleses en el siglo XVIII sobrevivían con una dieta más pobre que sus contemporáneos chinos. Obtenían un aporte adicional de calorías bebiendo cerveza y muchos de ellos apenas consumían pan en absoluto, sino harina o papilla de cebada y sopa de lentejas (Goldstone, 2002a, p. 26). Por otro lado, aunque puede que en 1800 los ingleses obtuviesen hasta un 4 por 100 de su consumo diario de calorías del azúcar del Nuevo Mundo, la cifra no resulta demasiado impresionante. Y es más que probable, además, que en el continente europeo existiesen reservas suficientes de tierra para continuar alimentando a la población urbana sin los aportes de ultramar, simplemente difundiendo la mejor tecnología y antes de que las rupturas técnicas de finales del siglo XIX —ante todo, la producción industrial de fertilizantes- permitiesen estabilizar y luego revertir el deterioro ecológico sin recurrir a métodos intensivos en trabajo ${ }^{19}$.

El argumento resulta más convincente cuando se refiere a la oferta de fibras naturales para la producción textil, que era, desde luego, mucho menos elástica. El cáñamo y el lino simplemente no podían reemplazar al algodón americano: ambos —especialmente el cáñamo- eran considerados fibras inferiores, ambos eran más difíciles de procesar, y, lo que es más importante, su cultivo era extremadamente intensivo en trabajo y consumía gran cantidad de fertilizantes. Por lo que respecta a la lana, la sustitución de las importaciones de algodón americano hubiese exigido, considerando la productividad media de las granjas modelo, casi 9 millones de acres en 1815 y más de 23 millones en 1830, una cifra superior al total de los pastos y tierras cultivables de Gran Bretaña. Cuando se incluye el algodón, la contribución de los "acres fantasmas" del Nuevo Mundo al desarrollo económico europeo resulta bastante impresionante, y no es fácil imaginar cómo hubiese podido obtenerse fibra en esas cantidades sin la herencia de la conquista y las "malas instituciones" de la colonia. De manera que es muy probable que la aventura colonial fuese realmente decisiva, que crease en verdad una diferencia crítica sin la cual el desarrollo industrial europeo simplemente no hubiese podido arrancar y sostenerse en sus estadios iniciales. ¿Por qué no?

19 Puede ser cierto, además, que el conjunto de Europa disfrutase todavía en el siglo XVIII de ciertas "ventajas del subdesarrollo" frente a China: probablemente existían más recursos sin utilizar capaces de sostener el crecimiento industrial precisamente por la presencia de más barreras institucionales a la mercantilización y el uso intensivo de la tierra, desde los pastos comunales a los bosques para empleo cinegético. 
Desde luego las posibles interconexiones entre la dinámica interna de las economías europeas y sus relaciones de poder con el resto del mundo no se limitan a lo que acabo de anotar, y en ningún caso es la historia del comercio atlántico la única instancia en que cabe argumentar que el ejercicio del poder político de los estados europeos, respaldado por una fuerza militar imbatible, contribuyó al "ascenso de Occidente" y, en el reverso de la moneda, al "declive del resto".

Es importante señalar que los hallazgos de la nueva historiografía cuestionan, por un lado, las relaciones simplistas entre las diversas variantes de la institucionalidad política de los Estados y el desempeño económico. Las viejas nociones de que los emperadores y burocracias de los "despóticos" estados orientales "gobernaban la economía - y por tanto la base fiscal - de un modo irracional, que podría representarse como predador, arbitrario y peculiarmente maligno para el crecimiento smithiano", se están quedando, por ejemplo, rápidamente obsoletas (O’Brien, 2003, p. 75). Pero, por otro lado, esa misma historiografía tiende, más allá de las diferencias en las reglas que estructuran el juego político, a reafirmar la importancia de la "mano visible" de los Estados, de su capacidad tanto para producir bienes públicos como para promover una inserción ventajosa de las economías locales en los mercados mundiales. El "despótico" estado Qing cumplió bien la primera tarea antes de colapsar en las sucesivas crisis políticas que jalonaron, sobre el trasfondo del creciente poder militar europeo, su catastrófico siglo XIX. Sin embargo, mucho antes de que se produjese aquel colapso, los estados de Europa occidental le habían ya ganado la mano en la construcción de una red mundial de intercambios sometida a sus intereses.

De modo que, hasta el momento, en el origen del crecimiento sostenido de la economía europea parecen combinarse dos lógicas distintas: por un lado, la emergencia gradual de unos mercados más eficientes; por otro, la construcción política de relaciones de comercio asimétricas en el seno de la economía mundial. No habría habido crecimiento preindustrial sin lo primero. Pero probablemente el desarrollo de la manufactura británica tampoco hubiera podido mantener su rumbo sin colonias, sin esa periferia "especial", institucional y políticamente configurada para exportar cantidades crecientes de productos intensivos en tierra de la que Gran Bretaña dispuso y China no. Desde luego, la nueva historiografía sobre el Oriente no va a conseguir que veamos en la empresa colonizadora "el" motor de la transición hacia el crecimiento sostenido de las economías europeas. Tampoco lo pretende. Pero puede que sí nos obligue a reconocer, con el propio Patrick O'Brien, autor en otro tiempo del que es tal vez el trabajo más citado por quienes cuestionan el valor económico de las colonias, que tampoco es fácil interpretarla como un proceso "periférico", ajeno por completo a las "auténticas causas" del "ascenso de Occidente"20.

20 Su artículo clásico es O’Brien (1982). El reconocimiento de que los hallazgos de la nueva historiografía obligan a reevaluar el papel de las colonias se encuentra en $\mathrm{O}^{\prime}$ Brien (2003), p. 79. 


\section{Los fundamentos intelectuales de la transformación productiva: los mercados y la autonomía de las ideas}

La explotación del carbón mineral en Inglaterra, más que al acceso a los "acres fantasmas" de América, suele disfrutar del título de hito clave en la ruptura de los límites ecológicos al crecimiento, dada la enorme ampliación de los recursos disponibles que supuso el tránsito de las economías "orgánicas" tradicionales a la economía "inorgánica" moderna (Wrigley, 1993). En el relato de Ken Pomeranz, el carbón ocupa también un lugar central en la explicación de la divergencia súbita entre los dos mundos económicos que hasta bien avanzado el siglo XVIII transitaban por sendas paralelas de desarrollo. Para él, la "anomalía" inglesa, la extraña trayectoria de la región de Europa que fue capaz de superar los márgenes naturales del crecimiento preindustrial, podría explicarse, en lo esencial, como una combinación "afortunada" de colonias y carbón.

"Afortunada", ya que, según su interpretación, lo decisivo en la construcción del segundo pilar de la divergencia no fue la superior capacidad técnica de los europeos frente a los chinos para hacer uso del carbón mineral, sino el mero azar geográfico. El hecho de que en Inglaterra existiesen abundantes depósitos de carbón fácilmente accesibles, mientras en China las minas se encontraban muy apartadas de las áreas más prósperas y pobladas, en lugares que enfrentaban unos problemas de transporte que sólo pudieron resolverse tras la aparición del ferrocarril. O el que las minas inglesas tuviesen, además, tendencia a inundarse a medida que se iban trabajando vetas de una cierta profundidad, mientras a las chinas les ocurría exactamente lo contrario: extremadamente secas, sufrían con frecuencia explosiones de gas. Por eso, si tanto China como Europa occidental disponían de la habilidad técnica y los conocimientos mínimos necesarios para desarrollar la máquina de vapor, sólo en Inglaterra se dieron las condiciones geográficas apropiadas para la explotación intensiva del carbón mineral y sólo allí el modelo primitivo de Newcomen tuvo la oportunidad de aplicarse al bombeo de agua en boca de mina, el único lugar donde el carbón era suficientemente barato como para que su empleo resultase racional pese a su terrible ineficiencia energética. De no haber sido por aquéllas afortunadas coincidencias, el ingenioso "invento" hubiese nacido muerto ${ }^{21}$.

Es muy posible que esta concatenación de factores casuales fuese realmente decisiva y que, en ausencia de aquellas condiciones geográficas peculiares, la trayectoria de nuestras economías hubiese sido muy distinta. Seguramente cualquier

21 Pomeranz (2000), pp. 61-68. Este mismo relato "azaroso" del desarrollo de la tecnología del vapor puede encontrarse ya en algún manual universitario escrito desde la perspectiva de la "historia mundial". Por ejemplo, en Marks (2002). 
"revolución industrial" contrafáctica, dirigida por las economías continentales y dependiente - además del lino y de la lana- de la tecnología hidráulica "francesa" y de la energía eólica "holandesa", no hubiese llegado demasiado lejos. Pero es fácil ver que el límite de este relato azaroso es que permanece necesariamente mudo sobre las razones de la prodigiosa aceleración del ritmo de la innovación técnica que siguió al primer conjunto de "invenciones" y permitió un aprovechamiento económico cada vez más eficiente del mundo de posibilidades abierto por la nueva fuente de energía. Porque lo que finalmente "transformó a la Revolución Industrial en la 'gran divergencia' fue la persistencia del cambio técnico después de la primera oleada" de invenciones (Mokyr, 2003, p. 19). El azar geográfico puede ayudarnos a entender en parte el arranque del proceso - por qué "ocurrió" aquella Revolución industrial一, pero no a explicarnos por qué se sostuvo más allá de, digamos, 1820. Y puede que ésta sea la pregunta verdaderamente importante (Mokyr, 2002, p. 31).

Llegados a este punto, la respuesta convencional de la historia económica consiste en apelar a la estructura de incentivos que creaban en las diversas economías las reglas que ordenaban el funcionamiento de los mercados. En un mercado abierto y con derechos de propiedad bien definidos y protegidos, los productores tienen un incentivo sistemático para adoptar cualquier innovación disponible capaz de mejorar sus rendimientos y, en ausencia de interferencias políticas, resulta simplemente imposible que los grupos amenazados por la introducción de una nueva técnica la detengan, dado que no pueden controlar lo que hacen los otros: en los mercados no cabe votar "no". Por otro lado, la variación en la intensidad de la oferta de innovaciones podría explicarse a partir de la mejor o peor definición y protección de los derechos de propiedad intelectual. Dado que los agentes responden a los incentivos económicos, cuanto más eficientes sean los sistemas de patentes, mayor será el ritmo de la invención.

Si todas las variantes del relato maestro del "milagro europeo" cuentan una historia auto-contenida que, como argumenté en el epígrafe anterior, nunca exigen trascender la dinámica interna de la propia Europa para explicar la divergencia, tenemos aquí una versión "estrecha" que propone un relato auto-contenido también en un segundo sentido, dado que no necesita ir más allá de la descripción de los cambios institucionales que impulsaron el desarrollo de los mercados que constituyen el objeto de la corriente principal de la economía. Por supuesto que hay mucho de cierto en este relato. No hay duda de que el marco institucional de la economía determina el modo en que se actualiza el potencial del conocimiento humano en forma de "técnicas" o "inventos", y desde luego la intensidad con que éstos se aplican a las actividades productivas. Pero la historia de la relación entre las ideas y el arranque de un proceso continuo de mejoras técnicas en la producción durante la Revolución Industrial parece bastante más compleja de lo que propone el relato institucionalista básico sobre los mercados y los derechos de propiedad. 
Joel Mokyr lleva un tiempo insistiendo en la idea de que la nueva dinámica del cambio técnico que se inició entonces sólo pudo sostenerse debido a la presencia de una base suficientemente amplia de conocimientos útiles, un fundamento epistémico sólido sin el que las "invenciones" sólo pueden ser - como había sido la norma hasta entonces- casualidades afortunadas que difícilmente dan lugar a mejoras ulteriores. Y si es posible que las características de las instituciones económicas expliquen bastante bien la selección de elementos en el stock de conocimientos disponibles y el ritmo de su aplicación a las actividades productivas, lo primero, el proceso mismo de generación de conocimientos sobre las regularidades de la naturaleza, no puede tratarse como una actividad empresarial más. Hume dijo en una ocasión que nunca faltarán libreros si hay gente dispuesta a comprar libros, pero que podría muy bien haber infinidad de lectores potenciales y no presentarse ningún buen autor. Ocurre, en términos del propio Mokyr, que la elaboración del menú y la selección de los platos por los comensales son problemas de diferente orden (Mokyr, 2002, p. 294).

Las motivaciones del pequeño ejército de pensadores que hicieron aportaciones clave en el amanecer de la ciencia moderna, por ejemplo, trascendieron sin duda lo puramente material. La vocación de servicio a la sociedad, la pasión por comprender la naturaleza o el reconocimiento de los pares, desempeñaron un papel crítico en sus afanes, y por eso no parece fácil ofrecer una explicación positiva de sus acciones y del proceso que desencadenaron. Seguramente la convivencia prolongada con las instituciones capitalistas de mercado y su estructura de incentivos pueda explicar el que "los intereses económicos hayan ido haciéndose crecientemente importantes a lo largo del último siglo y medio" para estimular y orientar el desarrollo científico (Mokyr, 2002, p. 287). Pero, si la dinámica del conocimiento mantiene hoy una menor autonomía frente a los incentivos económicos del mercado - lo que explicaría su mayor orientación práctica y, al menos en parte, la aceleración progresiva del ritmo de la innovación técnica-, ello no obsta para que en el origen dominasen otros mecanismos sobre los que la ciencia económica permanece muda. El mismo Mokyr recordaba en otro de sus trabajos la historia de Benjamin Thompson, uno de los muchos ejemplos del maridaje ilustrado entre la especulación teórica y las aplicaciones prácticas del conocimiento para "el servicio de la humanidad", quien, al igual que tantos otros, se negó a patentar ninguno de sus inventos porque, "como auténtico hijo de la Ilustración, estaba comprometido con el concepto de la libertad y gratuidad del conocimiento" (Mokyr, 2003, p. 34).

Parece entonces que la explicación nítida y elegante que ofrece el neoinstitucionalismo más afín a la economía neoclásica de la aceleración del cambio técnico en las primeras etapas de la revolución industrial, resulta profundamente insatisfactoria si empezamos a tomarnos en serio la idea de que la ampliación previa de la base epistémica de la innovación fue una condición necesaria para la continuidad de ésta y, por lo tanto, para la sostenibilidad del nuevo crecimiento. Y cuanto más en serio nos la 
tomemos, más nos veremos obligados a ir desbordando el relato auto-contenido sobre el desarrollo de la economía de mercado y el perfeccionamiento gradual de las instituciones que lo respaldan. Porque lo que tenemos delante es una transformación cultural de largo plazo que mantiene una relación bastante difusa con cualquier entorno institucional preciso, y desde luego con las instituciones propiamente económicas.

El cambio crítico podría describirse como un proceso que afectó al conjunto de Europa "occidental" desde la "revolución científica" del siglo XVII (Maddison, 2000, p. 4). O bien a partir de su participación colectiva en un movimiento de "Ilustración industrial" que, con su insistencia en la observación, comprensión y manipulación de las fuerzas naturales al servicio del progreso social, cabría interpretar como el puente entre la revolución científica y la industrial (Mokyr, 2002, p. 34). También cabe insistir, finalmente, en la importancia de los "estilos nacionales", haciendo hincapié en el hecho de que sólo en Gran Bretaña arraigó profundamente una cultura de "ciencia mecánica" experimental a finales del siglo XVII y comienzos del XVIII, mientras que en el resto de Europa la corriente se diluía o se detenía por completo a causa de la reacción de la Iglesia Católica o incluso de la Iglesia Reformada Holandesa (Jacob, 1997, p. 145). En este caso, el cambio crítico podría interpretarse como un giro o una variante cultural novedosa y específicamente británica, un desarrollo bastante "raro e insólito" dentro del tronco de la ciencia especulativa euroasiática (Goldstone, 2002b, p. 373).

Los historiadores de la economía han tendido a minusvalorar la importancia de los cambios en la escala del conocimiento, su difusión y su orientación, probablemente porque lo han identificado con la ciencia formal. Se ha señalado muchas veces que en las etapas iniciales de la revolución industrial los saberes prácticos atesorados por ingenieros, maestros metalúrgicos y toda una pléyade de artesanos ingeniosos fueron mucho más valiosos para la economía que la ciencia de un Cavendish, un Lavoisier o un Dalton (Mokyr, 2004, p. 25). Pero lo cierto es que el desarrollo de la "ciencia" fue sólo una parte - y tal vez una parte comparativamente pequeña- de los progresos de la base epistémica de la innovación técnica mediante la acumulación de conocimientos sobre las regularidades de la naturaleza y la ampliación de las oportunidades para acceder a ellos.

Científico o no, el conocimiento útil acumulado alcanzó en un determinado momento un nivel crítico desde el que resultaba posible el inicio de una retroalimentación constante entre las nuevas técnicas y el estudio de las regularidades naturales subyacentes. Si las técnicas funcionaban y no se entendía bien por qué, el misterio encerrado en su éxito estimulaba las investigaciones de científicos y filósofos naturales, y éstas a su vez impulsaban extensiones y perfeccionamientos progresivos de las técnicas. Por otro lado, los nuevos instrumentos para la observación, medición y procesamiento de materiales ofrecían nuevas posibilidades para la "revelación artificial" de las regularidades de la naturaleza, promoviendo la emergencia de nuevo 
conocimiento básico que eventualmente desencadenaba otros avances técnicos. Ocurrió así lo que la jerga de la teoría de sistemas describiría como un "cambio de estado" por el que la tecnología transitó de una dinámica dominada por los rendimientos decrecientes hacia otra en la que la interacción entre los avances en el conocimiento sobre el mundo natural y los desarrollos técnicos se alimentaban mutuamente en una suerte de "escalada" (Mokyr, 2004, pp. 27-28).

Cuando el conocimiento acumulado alcanzó el nivel en el que aquel "cambio de estado" podía ocurrir por primera vez, las instituciones capitalistas de Gran Bretaña ofrecieron un marco adecuado para el desarrollo sostenido de la industria. Pero de esto no se sigue que fuesen el único marco institucional posible, y mucho menos que aquellas instituciones creasen por sí mismas los saberes necesarios para que la transformación pudiese ocurrir. De manera que el mercado y las instituciones que respaldaron su desarrollo fueron tan sólo un hilo, y tal vez no el más importante, en la urdimbre de la historia del avance del conocimiento útil. Una historia en la que nada era ineluctable y que, como destaca el propio Mokyr, guardó un "alto grado de autonomía" respecto a la dinámica económica (Mokyr, 2002, p. 293). Una historia que, por lo mismo, no puede reducirse en ningún caso al desarrollo progresivo de la comercialización de la economía, tal como el pensamiento neoclásico y el neoinstitucionalismo más afín a él podrían llevarnos a imaginar.

\section{Conclusiones}

La búsqueda de "la" causa de la riqueza y la pobreza de las naciones, del "factor crucial" del crecimiento, tiene una historia larga, jalonada de esperanzas frustradas (Adelman, 2000). En los últimos años, la tendencia ha sido considerar que ese "factor crucial" son las instituciones que regulan el funcionamiento de los mercados. $\mathrm{Si}$ se adoptasen unas "buenas instituciones" capaces de crear los incentivos adecuados, el crecimiento sostenido vendría por sí mismo ${ }^{22}$. Y, como afirmé en el primer epígrafe de este trabajo, se trata de un conjunto preciso de instituciones, destilado de la experiencia histórica occidental, que se considera indiscutiblemente superior para promover el desarrollo económico.

Ahora bien, si nos atenemos a lo que hemos relatado en estas páginas, deberíamos conceder que lo que vamos sabiendo de la historia de otras civilizaciones, cuan-

22 Las políticas de desarrollo funcionarían si se produjese un sencillo retorno al "principio básico de la economía", que sostiene que "la gente responde a los incentivos (y) lo demás es nota a pie de página". Easterly (2003), p. XIV. 
do menos de la China moderna, parece apuntar a que el dinamismo mercantil es compatible con diseños institucionales bastante diversos. De manera que el descubrimiento del carácter tardío de la divergencia entre las regiones más avanzadas de Oriente y Occidente está contribuyendo, en primer lugar, a restar credibilidad al determinismo que subyace a las políticas del "monocultivo institucional". Si ya no estamos tan seguros como solíamos del carácter excepcional del desempeño europeo antes de la Revolución Industrial, tendremos menos razones para afirmar la superioridad indiscutible de los marcos institucionales que impulsaron el desarrollo de sus economías. Hubo multitud de diferencias en las instituciones que respaldaron y regularon el funcionamiento de los mercados en China y Europa, pero no parece fácil proponer una jerarquía nítida si resulta que no hubo una variación clara en los resultados en términos de crecimiento y bienestar.

Podemos pensar que, en este punto, los hallazgos de la historiografía revisionista están proyectando hacia el pasado el panorama institucional variado y complejo que caracteriza a las economías más exitosas en nuestro presente. El propio North, enfrentado al extraordinario rendimiento del "peculiar" modelo chino en el presente, ha terminado admitiendo que "las instituciones que han emergido en el mundo occidental, tales como los derechos de propiedad y los sistemas judiciales, no tienen por qué ser copiadas fielmente"; que la clave, a fin de cuentas, "es la estructura de incentivos que se crea, no la imitación de las instituciones occidentales" (North, 2005, p. 159). Y los nuevos datos sobre el desempeño de la economía china en el pasado que desmienten el mito del "excepcionalismo" europeo no hacen sino reforzar esta concesión al relativismo.

Pero, en segundo lugar, al defender el carácter tardío y abrupto de la divergencia, la nueva historiografía tiende también a destacar el papel decisivo que desempeñaron en el proceso factores causales distintos del marco institucional que regulaba el funcionamiento interno de cada una de las economías regionales. Es decir, que los diversos marcos institucionales que pueden crear incentivos adecuados para la actividad de los agentes económicos no ofrecen tampoco una explicación suficiente de la emergencia del crecimiento sostenido.

En el origen del crecimiento sostenido, por el contrario, se combinan al menos tres lógicas diferentes que no cabe reducir y que, según creo, tampoco tiene sentido intentar jerarquizar. En primer lugar, tenemos la emergencia gradual de unos mercados más libres y eficientes, producto de cambios institucionales que tuvieron lugar en el transcurso de la Edad Moderna tanto en Europa occidental como en la China Qing, con una cierta estabilidad política y seguridad de la propiedad. El crecimiento preindustrial fue producto de la mercantilización intensa impulsada por aquellos entornos institucionales favorables, algo que compartieron los dos extremos más avanzados de Eurasia y que fue sin duda una condición necesaria para cualquier progreso ulterior. Pero tenemos también, en segundo lugar, la construcción política 
de relaciones de intercambio asimétricas en el seno de la economía mundial en construcción y, finalmente, un salto radical en la tasa de innovación en la tecnología productiva que hubiese resultado imposible sin un largo proceso previo de acumulación de conocimiento útil y que tal vez no habría fructificado sin la emergencia en Gran Bretaña de una cultura peculiar de "ingeniería científica".

Estas tres lógicas - las del mercado, el poder político y el conocimiento- no pueden reducirse, aunque sin duda mantienen intensas relaciones mutuas. La expansión ultramarina de Europa no fue sólo expresión de su dinamismo mercantil, por mucho que no pueda explicarse sin éste. El estado británico desempeñó un papel fundamental en la transición hacia el crecimiento sostenido entre otras cosas porque tuvo la determinación y la capacidad necesarias — sus impuestos eran los más elevados de Europa en el siglo XVIII y su burocracia la más extensa- para lograr la hegemonía internacional. De modo que la violencia constante en el seno de un sistema interestatal fuertemente competitivo no fue importante únicamente, como pretende North, porque enseñó a algunos gobernantes el valor de proteger los derechos de propiedad y promover la lógica del mercado, sino también porque impulsó un tipo de estado activista y agresivo en el escenario internacional, mucho más poderoso que el "inhibido" estado chino. La acumulación de conocimientos útiles y la emergencia progresiva de una cultura de ciencia aplicada siguieron también una lógica autónoma, que no cabe reducir al avance de la mercantilización de la economía y los cambios institucionales que lo respaldaron. El proceso tuvo su propia historia, producto tanto de evoluciones culturales de larga data como del resultado "afortunado" de determinados conflictos políticos. Una vez que esa historia culminó, la cultura científica pudo rendir sus frutos en contextos institucionales diversos, incluso en los más hostiles a la libertad de pensamiento y la lógica de mercado, como demuestra de un modo espectacular la industrialización soviética.

Lo que la nueva historiografía tiende a sugerir en conjunto es, por lo tanto, que las razones del "ascenso" temporal de Europa no pueden hallarse en un único factor, sino "en la acumulación impredecible de muchas características", algunas de las cuales estuvieron presentes por separado, con mayor o menor intensidad, en otras partes del mundo que, sin embargo, no iniciaron la senda del crecimiento sostenido (Bayly, 2004, p. 71). Y esta insistencia en la causalidad compleja debería tener consecuencias para el modo en que pensamos y abordamos hoy las políticas de desarrollo. Podría argumentarse que a la postre todas las teorías del desarrollo son en alguna medida multicausales y que lo que en realidad se dirime en el debate son los énfasis, y con ellos las prioridades de la agenda política. Eric Jones, por ejemplo, piensa que de la historia podría derivarse "una ordenación lexicográfica de los determinantes del crecimiento en la que lo primero y más importante es la estabilidad política y la seguridad de la propiedad" (Jones, 2002, p. 12). Ahora bien, el problema con estas "ordenaciones lexicográficas" es que en el mundo social las entradas 
del diccionario suelen mantener relaciones de dependencia mutua, y esto implica que "el primer determinante" podría no mantenerse en el tiempo ni producir efectos apreciables si no se hacen presentes los demás. Los países en desarrollo necesitan sin duda estabilidad e instituciones que incentiven las actividades productivas; pero también soberanía, un espacio autónomo para elaborar sus propias prioridades políticas y promover una inserción favorable en la economía internacional; y, finalmente, recursos y determinación para crear la base de capacidades técnicas y las redes de innovación que impulsan el crecimiento económico moderno. Cuando menos, hasta la fecha todos los pueblos que han iniciado una ruta de convergencia con las economías más prósperas se beneficiaron en algún momento de algún equivalente de aquella acumulación simultánea de muchas características favorables que creó en su momento el "milagro europeo" original.

\section{Bibliografía}

ACEMOGLU, Daron; JOHNSON, Simon; y ROBINSON, James A. (2005): “The Rise of Europe: Atlantic Trade, Institutional Change and Economic Growth", The American Economic Review, 95 (3), pp. 546-579.

ADELMAN, Irma (2000): "Fallacies in Development Theory and Their Implications for Policy", en MEIER, Gerald M., y STIGLITZ, Joseph E. (eds.), Frontiers of Development Economics: The Future in Perspective, New York, Oxford University Press, pp. 103-134.

ALLEN, Robert C. (2003): "Productivity in Chinese and English Agriculture 16001800", comunicación presentada en All-UC Group in Economic History Conference, Irvine, Ca., 8-10 noviembre.

AMBROSOLI, Mauro (1997): The Wild and the Sown, Cambridge, Cambridge University Press.

BAYLY, Christopher A. (2004): The Birth of the Modern World, 1780-1914: Global Connections and Comparisons, Oxford, Blackwell.

BLAUT, James M. (1993): The Colonizer's Model of the World. Geographical Diffusionism and Eurocentric History, New York, The Guilford Press.

BRENNER, Robert (1989): "La base social del desarrollo económico", en ROEMER, John R. (ed.), El marxismo: una perspectiva analítica, México, Fondo de Cultura Económica, pp. 33-67.

CHAKRABARTY, Dipesh (2000): Provincializing Europe: Postcolonial Thought and Historical Difference, Ewing, Princeton University Press.

DE VRIES, Jan (1994): “The Industrial Revolution and the Industrious Revolution", The Journal of Economic History, 54 (2), pp. 249-270. 
-(2001): "The Industrious Revolutions in East and West", University of California at Berkeley, Diciembre, manuscrito inédito.

-(2003): "The Industrious Revolution and Economic Growth, 1650-1830", en DAVID Paul A., y THOMAS, Mark (eds), The Economic Future in Historical Perspective, London, Oxford University Press, pp. 43-71.

DE VRIES, Jan, y WOUDE, Ad van der (1997): The First Modern Economy: Success, Failure, and Perseverance of the Dutch Economy, 1500-1815, Cambridge, Cambridge University Press.

DIAMOND, Jared (1998): Armas, gérmenes y acero. La sociedad humana y sus destinos, Madrid, Debate.

EASTERLY, William (2003): En busca del crecimiento. Andanzas y tribulaciones de un economista del desarrollo, Barcelona, Antoni Bosch.

ESCOBAR, Arturo (1997): "Anthropology and Development", International Social Science Journal, 154, pp. 497-516.

EVANS, Peter (2004): “Development as Institutional Change: The Pitfalls of Monocropping and Potentials of Deliberation", Studies in Comparative International Development, 38 (4), pp. 30-53.

FERGUSON, James (1994): The Anti-Politics Machine: Development, Depoliticization, and Bureaucratic Power in Lesotho, Minneapolis, University of Minnesota Press.

FRANK, André Gunder (1998): ReOrient: Global Economy in the Asian Age, Berkeley, University of California Press.

GOLDSTONE, Jack A. (2002a): "Missing the Forest for the Trees: A Comparison of Productivity in Agriculture in Preindustrial England and Imperial China", http://aghistory.ucdavis.edu/goldstone.pdf.

—(2002b): "Efflorescences and Economic Growth in World History: Rethinking the 'Rise of the West' and the Industrial Revolution', Journal of World History, 13 (2), pp. 323-389.

HODGSON, Geoffrey M (2001): How Economics Forgot History: The Problem of Historical Specificity in Social Science, London, Routledge.

JACOB, Margaret (1997): Scientific Culture and the Making of the Industrial West, New York, Oxford University Press.

JONES, Eric L. (1991): El milagro europeo, Madrid, Alianza Editorial.

-(2002): The Record of Global Economic Development, Cheltenham, Edward Elgar.

LANDES, David S. (1999): La riqueza y la pobreza de las naciones, Barcelona, Crítica.

LECHNER, Norbert (1986): La conflictiva y nunca acabada construcción del orden deseado, Madrid, CIS.

LEE, James Z., y FENG, Wang (1999): One Quarter of Humanity. Malthusian Mythology and Chinese Realities, 1700-2000, Cambridge, Harvard University Press.

LEVINE, David (2000): At the Dawn of Modernity, Berkeley, University of California Press. 
LITTLE, David (1991): Varieties of Social Explanation. Introduction to the Philosophy of Social Science, Boulder, Westview Press.

MADDISON, Angus (2000): “Economic Progress: The Last Half Century in Historical Perspective", en CASTLES, Ian (ed.), Facts and Fancies of Human Development, Camberra, Academy of the Social Sciences in Australia, pp. 1-22.

MARKS, Robert B. (2002): The Origins of the Modern World. A Global and Ecological Narrative, Lanham, Rowman and Littlefield.

MOKYR, Joel (2002): The Gifts of Athena, Princeton, Princeton University Press.

- (2003, en prensa): "Long-Term Economic Growth and the History of Technology", en AGHION, Philippe, y DURLAUF, Steven, Handbook of Economic Growth, London, Elsevier.

-(2004): Useful Knowledge as an Evolving System: The View from Economic History, Papers on Economics \& Evolution, 0423, Jena, Max Planck Institute for Research into Economic Systems.

NORTH, Douglass C. (1993): The Paradox of the West, Economics Working Paper Archive at WUSTL.

-(2005): Understanding the Process of Economic Change, Princeton, Princeton University Press.

NORTH, Douglass C., y THOMAS Robert P. (1978): El nacimiento del mundo occidental. Una nueva historia económica (900-1700), Madrid, Siglo XXI.

O’BRIEN, Patrick K. (1982): “European Economic Development: The Contribution of the Periphery", Economic History Review, 35 (1), pp. 1-18.

-(2003): "The Deconstruction of Myths and Reconstruction of Metanarratives in Global Histories of Material Progress", en STUCHTEY, Benedikt, y FUCHS, Eckhardt, Writing World History 1800-2000, Oxford, Oxford University Press.

PARTHASARATHI, Prasannan (2001): The Transition to a Colonial Economy: Weavers, Merchants and Kings in South Asia, 1720-1800, Cambridge, Cambridge University Press.

POMERANZ, Kenneth (2000): The Great Divergence. China, Europe, and the Making of the Modern World Economy, Princeton, Princeton University Press.

RODRIK, Dani (2003): "Growth Strategies", Harvard University, septiembre, http:// ksghome.harvard.edu/ .drodrik.academic.ksg)

SKIDELSKY, Robert (2001): "The Mystery of Growth", The New York Review of Books, 50 (4), 13 de marzo, pp. 28-31.

SZTOMPKA, Piotr (1995): Sociología del cambio social, Madrid, Alianza Editorial.

TAYLOR, Alan (1998): "Frank Versus Landes", http://www.eh.net/lists/archives/eh.res/ may-1988/

VRIES, Peer H. H. (2001): “The Role of Culture and Institutions in Economic History. Can Economics Be of any Help?", NEHA-Jaarboek, 64, pp. 28-60.

WRIGLEY, Edward A. (1993): Cambio, continuidad y azar, Barcelona, Crítica. 